\title{
Melioidosis: An Emerging Infection in Bangladesh
}

(BIRDEM Med J 2016; 6(2): 63-66)

Melioidosis is an infectious disease caused by a gramnegative bacterium, Burkholderia pseudomallei, found in soil and water. It is of public health importance in endemic areas. Whitmore first described it in Burma hence it is also named as Whitmore disease.

Melioidosis is an emerging infection in Bangladesh. The wide range of clinical presentations include severe community-acquired pneumonia, septicaemia, central nervous system infection and less severe soft tissue infection. Diagnosis depends heavily on the clinical microbiology laboratory for culture. Burkholderia pseudomallei, the bacterial cause of melioidosis, is easily cultured from blood, sputum and other clinical samples. However, B.pseudomallei can be difficult to identify reliably, and can be confused with closely related bacteria, some of which may be dismissed as insignificant culture contaminants. Serological tests can help to support a diagnosis of melioidosis, but by themselves do not provide a definitive diagnosis. The use of a laboratory discovery pathway can help reduce the risk of missing atypical B. pseudomallei isolates. Recommended antibiotic treatment for severe infection is either intravenous Ceftazidime or Meropenem for several weeks, followed by up to 20 weeks oral treatment with a combination of trimethoprim-sulphamethoxazole and doxycycline. Consistent use of diagnostic microbiology to confirm the diagnosis, and rigorous treatment of severe infection with the correct antibiotics in two stages; (acute and eradication), will contribute to a reduction in mortality from melioidosis.

\section{Signs and symptoms}

Melioidosis has a wide range of signs and symptoms that can be mistaken for other diseases such as tuberculosis or more common forms of pneumonia. Multiple site infection, abscess formation, longer time infection mimics tuberculosis.

1. Localized Infection: Localized pain or swelling, fever, ulceration, abscess

2. Pulmonary Infection: Cough, chest pain, high fever, headache, anorexia
3. Blood stream infection: Fever, headache, respiratory distress abdominal discomfort, joint pain, muscle tenderness, disorientation

4. Disseminated Infection: Fever, weight loss, stomach or chest pain, muscle or joint pain, headache, seizure

\section{Microbiology}

Melioidosis, also known as Whitmore disease, is caused by the bacterium Burkholderia pseudomallei (a motile, aerobic, non-spore-forming bacillus). It is clinically similar to glanders, although the epidemiology differs. The bacteria thrive in tropical climates. The disease is endemic in Southeast Asia and Australia, and is also found in the Middle East, India, and China (essentially tropical areas between latitudes 20 degrees north and south). Sporadic melioidosis cases have been reported in the United States . However, the worldwide incidence appears to be rising as a result of increased travel and epidemiological sophistication. Both humans and other susceptible animals may contract the disease.

Organisms are considered potential biological warfare agents, especially in the aerosolized form. In fact, this because they are highly infectious especially by inhalation and because of their resistance to routine antibiotics, it is easily available in tropics, fairly easy to cultivate, has high potential to become bacteremic, thereby increasing morbidity and mortality. Incubation period in naturally occurred infection can very from days to month to year. Incubition period after an aerosol attack is expected to be from 10-14 days. The bacteria have been classified as category B priority pathogens of bioterrorism by the National Institutes of Health and the Centers for Disease Control and Prevention (CDC).

\section{Pathophysiology}

Melioidosis is an infectious disease caused by $B$ pseudomallei (formerly P pseudomallei). The organism is distributed widely in the soil and water of the tropics. It is spread to humans through direct contact with a contaminated source, especially during the rainy season. 
The disease usually occurs in the fourth and fifth decades of life, especially among those who have chronic comorbidities such as diabetes, alcoholism, immunosuppression, and renal failure.

\section{Localized form}

Bacteria enter the skin through a laceration or abrasion, and a local infection with ulceration develops. The incubation period is 1-5 days. Swollen lymph glands may develop. Bacteria that enter the host through mucous membranes can cause increased mucus production in the affected areas.

\section{Pulmonary form}

When bacteria are aerosolized and enter the respiratory tract via inhalation or hematogenous spread, pulmonary infections may develop. Pneumonia, pulmonary abscesses, and pleural effusions can occur. The incubation period is 10-14 days. With inhalational melioidosis, cutaneous abscesses may develop and take months to appear.

\section{Septicemia}

With melioidosis, bacteremia is observed with chronically ill patients (eg, patients with HIV, patients with diabetes). They develop respiratory distress, headaches, fever, diarrhea, pus-filled lesions on the skin, and abscesses throughout the body. Septicemia may be overwhelming, with a $90 \%$ fatality rate and death occurring within 24-48 hours.

\section{Chronic form}

The chronic form involves multiple abscesses, which may affect the liver, spleen, skin, or muscles. . Melioidosis, in addition to this chronic form, can become reactive many years after the primary infection.

\section{Epidemiology}

Melioidosis is endemic in Southeast Asia and Australia. Hyperendemic in northern Thailand and northern Australia. Literature study suggests occurrence of sporadic melioidosis throught the globe.

In India, melioidosis has acquired the status of a newly emerging infectious disease.

\section{Mortality}

Melioidosis has had a reported mortality rate up to $90 \%$ if disseminated septicemia is present. In Australia, the mortality rate is $19 \%$, whereas in Thailand it is $50 \%$. It is most widespread in Thailand, where in one hospital, it was responsible for $19 \%$ of community-acquired sepsis and $40 \%$ of deaths from community-acquired septicemia. The fatality rate of melioidosis is greater in people with specific comorbidities, such as diabetes mellitus, renal dysfunction, or chronic pulmonary disease, and in people who are immunosuppressed for one reason or another.

\section{Clinical features History}

Melioidosis is transmitted to humans through direct skin contact with contaminated soil or water. Ingestion of contaminated water and inhalation of dust contaminated with the organism are other mechanisms of transmission. Cases of human-to-human transmission are rare but have been documented.

Generalized symptoms include fever, rigors, night sweats, myalgia, anorexia, and headache. Additional symptoms, which are based on the route of exposure, include chest pain, cough, photophobia, lacrimation, and diarrhea.

There is nothing specific about the presentation of these diseases and a strong clinical suspicion is required to assist in making a diagnosis in endemic regions. This is particularly true for patients with predisposing comorbidities, such as diabetes mellitus, chronic renal failure, alcoholism or malignancy; those who are immunosuppressed as the result of either diseases or drug treatment; and those living in or with a history of travel to endemic areas. However, in the event of an intentional bacteriological attack in other regions of the world, the above subsets of patients will be the most affected.

\section{Physical}

Physical findings may include fever, cervical adenopathy, hepatomegaly, or splenomegaly, and skin lesions including the following:.

- Severe urticaria has been reported during primary melioidosis

- During septicemia, flushing, cyanosis, and a disseminated pustular eruption can be seen. Pustules often are associated with regional lymphadenitis, cellulitis, or lymphangitis 
- Rarely, ecthymagangrenosum-like lesions and cutaneous abscesses (that sometimes ulcerate) may develop

Specifically, in melioidosis septicemia, high fevers and rigor are present. These findings may be accompanied by confusion, dyspnea, abdominal pain, muscle tenderness, pharyngitis, diarrhea, and jaundice. While the typical foci in these severe cases begin from the skin or the lungs, metastasis (liver, spleen, kidney, brainstem, parotid gland) will occur, leading to acidosis, shock, and death within 48 hours of presentation.

\section{Laboratory Studies}

Basic laboratory tests include the following:

A.Complete blood cell count (CBC) B.Liver function studies C.Gram stain and culture of blood, sputum, urine, and skin lesions. The organism is not a commensal so any specimen giving the growth is taken as diagnostic.

The CBC may reveal a mild leukocytosis with a left shift or leukopenia. Elevated liver enzyme levels may signify hepatic abscess formation.

In melioidosis specifically, laboratory studies may demonstrate anemia, leukocytosis, hepatic impairment, renal insufficiency, and coagulopathy.

Gram stain may reveal small, gram-negative bacilli, which stain irregularly with methylene blue or Wright stain, and they may demonstrate a safety pin bipolar appearance. Blood culture results may be negative. The median time for growth is 48 hours. In septicemic melioidosis, blood culture results may be negative until just before death. Meat nutrient agar or the addition of $1-5 \%$ glucose may accelerate growth of bacteria. Another useful culture medium for $B$ pseudomallei is Ashdown's selective medium.

Other tests include: a.Agglutination tests: Agglutination test results may be positive after 7-10 days, but a high background titer found in normal sera makes interpretation difficult. b.Indirect hemagglutination tests c. Polymerase chain reaction assays d.Immunofluorescence assays e.Enzyme immunoassays f.Complement fixation tests

Complement fixation tests are more specific and are considered positive for glanders if the titer is $1: 20$ or greater. A 4-fold increase in the titer for melioidosis is considered positive.

\section{Imaging/Studies}

Chest radiography may demonstrate bilateral bronchopneumonia, miliary nodules, segmental or lobar infiltrates, and cavitating lesions. With melioidosis, an abnormal chest radiography finding is present in up to $80 \%$ of patients (usually diffuse nodular shadowing).

Ultrasonography and computed tomography may reveal multiple, small discrete abscesses in both the liver and the spleen. These findings are highly suggestive of visceral melioidosis.

Bone and soft tissue musculoskeletal involvement may be seen with plain radiographs and magnetic resonance imaging. These findings are consistent with disseminated melioidosis.

\section{Management \\ Medical Care}

Limited information exists about antibiotic therapy melioidosis in humans because clinical studies examining antibiotic effectiveness in vivo are rare.

A. For localized disease, a 60- to 150-day course of oral amoxicillin/clavulanate, doxycycline, or trime thoprim/sulfamethoxazole (TMP-SMX) may be used.

B. For local disease with mild toxicity, combine two of the three regimens for 30 days, then switch to monotherapy with amoxicillin/clavulanate or TMPSMX for 60-150 days.

C. For extra-pulmonary suppurative disease, prolong treatment for 6-12 months. Drain abscesses surgically.

D. For severe and/or septicemic disease, initiate parenteral therapy for 2 weeks followed by oral therapy for 6 months (ceftazidime combined with TMP/SMX $8 \mathrm{mg}$ TMP/kg/d and $40 \mathrm{mg} \mathrm{SMX/kg/d}$ divided qid).

Alternative choices in severe cases of melioidosis include imipenem-cilastatin or meropenem with or without trimethoprim/sulfamethoxazole. Time period may need to be extended.If fever does not subside in two weeks without suspending the antibiotic should be continued

Administration of these drugs may continue for up to 4 weeks, depending on clinical response. Then, a 20-week course of doxycycline and TMP/SMX, which is said to 
minimize the likelihood of relapse better than amoxicillin/clavulanate, is administered.

The prevention of relapse in melioidosis is critical since it has been reported to occur in $23 \%$ of cases. Hence, the rationale behind the prolonged therapy. Other antibiotics with activity against Burkholderiapseudomallei include ceftriaxone, ticarcillin-sulbactam, and aztreonam.

Chemoprophylaxix:Currently, no proven preexposure or postexposure prophylaxis is available. Postexposure prophylaxis with TMP/SMX may be attempted. No vaccine is available for human glanders or melioidosis.

For acute human melioidosis, the most commonly recommended treatment regimen consists of ceftazidime or a carbapenem followed by TMP/SMX. For moderate to severe melioidosis, a treatment protocol from the Royal Darwin Hospital in Australia consists of the following:

- Ceftazidime $2 \mathrm{~g}$ IV q6h $(50 \mathrm{mg} / \mathrm{kg}$ up to $1 \mathrm{~g}$ in children) $\boldsymbol{o r}$

- Meropenem $1 \mathrm{~g}$ IV q8h $(25 \mathrm{mg} / \mathrm{kg}$ up to $1 \mathrm{~g}$ in children) plus

- Cotrimoxazole 320/1600 mg PO/IV BID (8/40 mg/ $\mathrm{kg}$ up to $320 / 1600 \mathrm{mg}$ in children).

This regimen is given for at least 14 days, but may have to be continued for longer. Once the acute episode is resolved, then the eradication period would commence.

\section{Investigational therapy}

BAL 30072, an investigational intravenously administered monosulfactam antibiotic, shows excellent activity against $B$ pseudomallei. Another promising avenue of therapy is inhalational immunotherapy with cationic liposome DNA complexes (CLDC). These complexes are potent activators of innate immunity within the pulmonary system, which makes this modality an attractive approach to inhalational exposure to these bacteria.

\section{Prevention}

A. Biosafety level 3 containment practices are required for laboratory staff.

B. In areas where melioidosis is endemic, persons who have chronic illnesses that lead to an immunocompromised state should avoid contact with soil and standing water. Wearing boots and gloves during agricultural work is advised.

C. No vaccine is currently approved for melioidosis. the research for a viable vaccine (eg, live-attenuated, inactivated whole cell, recombinant subunit) continues

\section{Prognosis}

- Untreated patients with septicemia have fatal outcomes. Before antibiotics, the death rate for septicemic disease was $95 \%$. It is greater than $50 \%$ for septicemic disease and $20 \%$ for localized disease despite treatment. Overall, the mortality rate is $40 \%$.

\section{Melioidosis in Bangladesh}

First case was published in Bangladeshi journal in 2001.The organism was detected in Gazipur soil in 2013. Since then Bangladesh is the $18^{\text {th }}$ definite country of Melioidosis in world.So far 31 cases has been reported in Bangladesh.Our case were detected through a pathway of microbiological analysis.Majority of them were chronic cases with multiple site infection provisionally diagnosed as disseminated TB as cause of prolonged fever and were on empirical anti TB drug.All of them were treated with initial IV high dose of Ceftazidime for 2-4 weeks followed by the eradication phase with combination of Co Trimoxazole and Doxicycline for minimum 6 months.

Our cases were collected through study of Pub Med and other literatures,hospital registry and personal communication.First case was a chance diagnosis like many others as revealed by literature.It was first treated as community acquired pneumonia seeing the lung lesion,then was on empirical ant-TB as he developed right knee arthritis, abscess over left shoulder and left chest wall. The first Microbiology sample was discarded as contamination.It was clinician's interest and experience(gained in Malyasia)of microbiologist which helped to clinch the diagnosis ending in successful outcome of treatment.

28 of our patients were diabetic,majority are cultivator. Soil of the district of Tangail, Mymensingh, Gazipur, Sylhet appear to have B. pseudomelli and hyperendemic for Melioidosis.

It is difficult to accept that we have only 31 cases over so many years in a country of heavy rainfall with a population of 160 million. Physicians, Microbiologists and people at large should be acquainted and remain survillent for this emerging new infectious disease of Melioidosis in our country. 